\title{
À la recherche de faux sauniers aux limites du Maine, de l'Anjou et de la Bretagne, à la fin du XVIIIe siècle
}

\section{Melaine Lefeuvre}

\section{(2) OpenEdition}

\section{Journals}

Édition électronique

URL : http://journals.openedition.org/abpo/1550

DOI : 10.4000/abpo.1550

ISBN : 978-2-7535-1487-4

ISSN : $2108-6443$

\section{Éditeur}

Presses universitaires de Rennes

\section{Édition imprimée}

Date de publication : 20 septembre 2002

Pagination : 19-31

ISBN : 978-2-86847-768-2

ISSN : 0399-0826

\section{Référence électronique}

Melaine Lefeuvre, " $\grave{A}$ la recherche de faux sauniers aux limites du Maine, de l'Anjou et de la Bretagne, à la fin du XVIIle siècle ", Annales de Bretagne et des Pays de l'Ouest [En ligne], 109-3 | 2002, mis en ligne le 20 septembre 2004, consulté le 01 mai 2019. URL : http://journals.openedition.org/abpo/1550 ; DOI : 10.4000/abpo. 1550 


\title{
À la recherche de faux sauniers aux limites du Maine, de l'Anjou et de la Bretagne, à la fin du XVIII ${ }^{\mathrm{e}}$ siècle $^{1}$
}

\author{
Melaine LEFEUVRE \\ Étudiant en histoire et patrimoine, université du Maine
}

"Interogé de son nom, surnom, âge, qualitté, demeure et relligion,

a répondu se nommer Pierre Boisramé, tisserand, âgé d'environ vingt trois ans, natif de la paroisse de Cossé demeurant dans ladite paroisse et être de la relligion catholique, apostolique et romaine ${ }^{2}$."

Voilà en général le peu d'informations personnelles que l'on peut obtenir lors du procès d'un petit criminel, a fortiori s'il s'agit d'un faux saunier. Au-delà de cette simple déclaration, souvent de pure forme, on peut être tenté d'en savoir plus sur un individu dont le passage devant les instances judiciaires de la ferme des gabelles ne nous révèle pas grand-chose de la vie et des antécédents. Pour des populations criminelles aussi particulières que celle des faux sauniers de la frontière de Bretagne, populations que l'on peut retrouver en très grand nombre dans les archives judiciaires, il était intéressant de pousser un peu plus loin la connaissance de leur situation économique ou de leurs relations sociales.

Pour cela, une recherche a été menée à partir des archives des matricules du bagne de Brest ${ }^{3}$ où une bonne partie des récidivistes ont été envoyés dans la seconde moitié du XVIII ${ }^{\mathrm{e}}$ siècle, le but étant de confronter les données obtenues dans les registres du bagne avec celles que l'on peut glaner dans les archives de la région d'origine des condamnés. L'originalité

1. Cet article rend compte d'un mémoire de maîtrise soutenu en juin 2001 à l'Université de Rennes 2 et dirigé par Annie Antoine. Je remercie aussi Philippe Jarnoux qui a suggéré ce mémoire et m'a aidé de ses remarques et conseils pertinents.

2. AN, commission de Saumur, Z1A/1110, Affaire Pierre Boisramé, interrogatoire du 23 août 1759 .

3. Service historique de la Marine, Brest, Série 207 à 2 O 15. Sur ces archives, cf. JoAnNIC-SETA, Frédérique, Le Bagne de Brest, 1749-1800, L'émergence d'une institution carcérale au siècle des Lumières, Rennes, 2000 et JARnOUX, Philippe, "Le Roi, la frontière et le contrebandier : les faux sauniers des confins de la Bretagne au bagne de Brest (17491776) ", actes du colloque international de Brest Violence et société en Bretagne et dans les pays celtiques, 18-20 mars 1999, Kreiz 13, 2000, p. 463-488. 
de la méthode de travail consiste à ne pas chercher dans les archives de justice des données statistiques ou des récits ponctuels mais à y poursuivre des parcours individuels. Ceci sera l'occasion d'interrogations épistémologiques et méthodologiques.

L'autre intérêt de ce type de recherche sur les faux sauniers vient du fait qu'ils ont déjà été très étudiés et sont donc assez biens connus, ce qui va permettre de confronter les résultats de cette enquête avec ceux qui ont été obtenus par les auteurs des recherches macro-historiques ${ }^{4}$. De plus, ces populations de bagnards ayant des origines géographiquement concentrées, elles sont donc théoriquement plus faciles à retrouver dans des archives locales.

Mais il faut bien noter que le but de cet article n'est pas de refaire l'histoire du faux saunage " par le bas ", mais plutôt d'avoir un autre point de vue sur des individus habituellement perçus d'une tout autre façon par les sources judiciaires et par les études sur les criminels.

\section{À la recherche de traces : de sources et de méthodes}

\section{Problèmes de sources : où les trouver?}

Le moyen d'une telle recherche c'est de croiser plusieurs sources d'origine différente concernant un individu de façon à pouvoir connaître les différentes facettes de son existence. L'idéal serait donc d'obtenir suffisamment d'informations sur chaque individu retrouvé dans le matricule du bagne de Brest afin d'aborder le personnage par plusieurs angles de vue différents de ceux que fournissent les archives pénitentiaires.

Le premier objectif était de vérifier et de compléter les informations individuelles contenues dans les registres du bagne. Dans un premier temps, il a fallu vérifier les conditions dans lesquelles le faux saunier avait été envoyé au bagne, c'est-à-dire retrouver la procédure de jugement qui s'est d'abord déroulé au grenier à sel ou devant d'autres juridictions des fermes des gabelles. Malheureusement, quand cette procédure a été retrouvée, elle nous renseignait bien mal sur l'individu auquel on avait à faire, car, contrairement aux archives judiciaires traditionnelles, les procédures des greniers à sel, comme celles de la commission de Saumur, informent particulièrement peu sur la personnalité de l'accusé, sur son entourage et ses activités. Ceci tient à leur brièveté, à l'absence de témoins, et au fait que les interrogatoires sont uniquement centrés sur l'obtention de l'aveu, celui-ci étant le plus souvent rapidement obtenu car le procès verbal se fait

4. Cf. les travaux de DuRAND, Yves, " La Contrebande du sel au XVIII ${ }^{\mathrm{e}}$ siècle aux frontières de la Bretagne, du Maine, et de l'Anjou ", Histoire Sociale, Revue Canadienne, 74, 1974, p. 227-269; HuvET-MarTinEt, Micheline, " La Répression du faux saunage dans la France de l'Ouest et du Centre à la fin de l'Ancien-Régime (1764-1789) ", $A B P O, \mathrm{n}^{\circ}$ 84, 3, 1977, p. 423-443 et "Faux saunage et faux sauniers dans la France de l'Ouest et du Centre à la fin de l'Ancien-Régime (1764-1789) ", $A B P O$, n $^{\circ} 85,1978,3$, p. 377-400 et 4, p. 573-594; ainsi que JARNOUX, Philippe, " Le Roi, la frontière et le contrebandier... ", art. cit. 
après un flagrant délit. Bref, rien de ce qui fait la richesse des archives judiciaires pour l'histoire sociale n'est présent ici. Ces procédures peuvent néanmoins donner quelques informations sur le dernier lieu d'habitation du prévenu, sur un de ses proches, ou sur sa situation familiale au moment de son arrestation. Elles suggèrent de poursuivre les recherches dans les archives fiscales : mais là encore, il y a très peu d'informations sur les faux sauniers, car ils font souvent preuve d'une très grande instabilité géographique. De même qu'ils évitent de payer la gabelle, ils rechignent à payer taille et/ou capitation. Restait à tenter de les découvrir dans les registres paroissiaux en se livrant à un travail méticuleux de généalogiste, mais ce travail est rendu difficile par les déplacements de ce type de population - d'ailleurs souvent célibataire - de paroisse en paroisse, souvent de part et d'autre de la frontière de Bretagne. Finalement, ce sont les archives notariales dont le dépouillement a été le plus fructueux : certes, le faux saunier, à la limite de la marginalité, passe peu devant le notaire, mais lorsqu'on a la chance de lui attribuer un acte avec certitude, alors c'est tout un flot d'informations qui nous est donné, ainsi qu'un certain nombre de renvois vers de nouveaux documents. La source idéale dans ce genre de recherche, c'est celle qui renvoie à une autre source, de façon à créer une chaîne de documents et ainsi un corpus de données suffisantes sur un individu. Il faut entendre par efficacité de la source l'aptitude de celle-ci à nous fournir un maximum d'informations, mais aussi l'indication d'autres documents concernant le faux saunier recherché.

\section{Problème de méthode : comment les trouver?}

Cette approche des repris de justice par le détail des parcours individuels est suffisamment rare pour autoriser quelques remarques sur la méthodologie qui a été mise en œuvre au cours de ce travail et qui se rattache à la fois à la prosopographie ${ }^{5}$ et à la micro-histoire ${ }^{6}$. Mais ni l'une ni l'autre ne donne de mode de recherche clef en main pour aborder des petits criminels dans des archives telles que celles-ci existent dans l'Ouest de la France. En fait, au fur et à mesure des premières investigations et de la mise en évidence des rares sources disponibles, la méthode a dû être mise au point progressivement en s'adaptant à la plus ou moins grande pauvreté des sources. Pour cela, il fallait partir des noms des individus pour lesquels on disposait du plus de renseignements, c'est-à-dire ceux dont on pouvait retrouver le procès qui les avait envoyés au bagne, soit l'archive la plus complète et la plus sûre à leur sujet. De cette source, on pouvait tirer des indices dirigeant ensuite la recherche vers d'autres documents relativement sûrs : l'indication d'un lieu d'habitation précis au sein d'une paroisse

5. BULST, Neithard, " Objet et méthode de la prosopographie », GENET, Jean-Philippe et LOTTES, Günther (dir.), L'État moderne et les élites, XIII -XVIII siècles, apports et limites de la méthode prosopographique, actes du colloque international CNRS-Paris 1, 16-19 octobre 1991, Paris, Publications de la Sorbonne, 1996, p. 467-482.

6. Ginzburg, Carlo et Poni, Carlo, "La Micro-histoire », Le Débat, 17, 1981, p. 133-136. 
peut orienter vers un rôle de taille ou éventuellement vers un bail signé quelques années plus tôt; l'allusion à une éventuelle récidive peut orienter vers une autre procédure judiciaire éventuellement plus complète. Et chaque nouveau document peut ouvrir lui-même sur une autre source, d'autres traces, et ainsi de suite. Évidemment, " il devient alors nécessaire de procéder plus à tâtons, en se fiant au hasard et à la chance ${ }^{7}$ ". Certains diront que cette recherche peut devenir du même coup hasardeuse, ce qui a pu être reproché aux études nominales, ne s'intéressant qu'aux individus. Mais justement, ce hasard peut être revendiqué comme aide à la recherche : la chance n'a-t-elle pas aidé bon nombre de grandes recherches historiques?

\section{L'exemple de deux faux sauniers}

$\mathrm{Au}$ bout du compte, les résultats de cette étude sont relativement modestes. Sur les 61 faux sauniers relevés sur la liste du bagne - soit 71 cas de faux saunage, en comptant les récidives - on a retrouvé 25 procédures, identifié formellement 6 individus dans les rôles de taille et de capitation et recueilli 17 actes notariés les concernant directement. Pour 10 faux sauniers, on a pu croiser au moins deux sources. Or, c'est justement ce croisement de sources concernant un individu qui est à la base de la démarche de la micro-histoire, car c'est l'unique moyen de pouvoir capter un individu en dehors du prisme forcément déformant d'une source unique.

Le bilan pourrait apparaître décevant si on n'avait pu réunir des informations un peu plus complètes pour quelques individus, quatre exactement, présents sur la liste. Il s'agit essentiellement de documents un peu plus riches que les autres qui permettent d'éclairer les débuts de leur vie. Le parcours de deux hommes, André Rousseau et Pierre Boisramé sera développé ici. Ils sont d'autant plus intéressants qu'ils pourraient être l'un et l'autre révélateurs de catégories d'individus pratiquant la contrebande, catégories qui avaient déjà été repérées par d'autres études sur le faux saunage. C'est pourquoi une étude attentive et détaillée de ces deux trajectoires peut se révéler utile pour une connaissance du faux saunage.

\section{André Rousseau}

On possède très peu de traces judiciaires pour André Rousseau, en dehors de son matricule à Brest, où il est allé deux fois. Du procès subi devant le grenier à sel de Craon, il ne reste plus rien. De son procès devant les Traites et gabelles de Vitré puis au Parlement de Bretagne, il ne reste plus qu'un interrogatoire et une fraction de déclaration de sentence. Néanmoins, cet interrogatoire nous apprend que, malgré le peu de décla-

7. GinZBURG, Carlo et PonI, Carlo, « La Micro-histoire », art. cit., 1981, p. 134. 
rations d'André Rousseau sur son propre compte, celui-ci fut arrêté pour la première fois le 18 mai 1764 " dans la Lande de Creuillière, près du village de la Gestrie, paroisse de Balots [sic] ", conduisant un cheval chargé de sel, et trois jours plus tard, qu'il est condamné à 300 livres d'amende pour faux saunage à cheval. Il ne doit pas avoir été capable de régler cette amende car il est envoyé, après conversion de la peine, pour une période de trois ans au bagne de Brest. Dans ce dernier procès à Vitré, il est en prison depuis trois mois, ayant été arrêté le 24 mai 1769 à la Richardière, paroisse du Pertre, pour faux saunage à col, c'est-à-dire pratiqué à pied. Son procès aurait dû être beaucoup plus rapidement mené, car il est condamné dès le 27 mai suivant à 500 livres d'amende, tarif habituel prévu en Bretagne pour de la simple contrebande de sel. Comme il l'avait fait la première fois, il reconnaît le délit immédiatement sans aucune contestation. Mais cette fois-ci, il a été enregistré sous une fausse identité, celle de Michel Rousseau, et on ne le reconnaît comme un récidiviste qu'au mois d'août suivant. Selon lui, « il déclare aux employés [de la Ferme] se nommer André Rousseau et non Michel Rousseau, les employés le connoissoient à suffire pour ne pas se laisser tromper ${ }^{8}$ ". Son procès a donc dû être réglé à l'extraordinaire, en raison de cette récidive, et il n'est définitivement clos par une sentence que le 21 août 1769. Il est alors condamné à six ans de galères et pour faux saunage en récidive et 300 livres d'amende. Si on se contente de ces informations que nous fournit la procédure judiciaire, on sait alors peu de chose sur le personnage. Il semble miséreux, incapable de payer la moindre amende, habitué de la contrebande. Mais si on remonte ne serait-ce que dix années auparavant, on peut lui imaginer un tout autre destin.

André Rousseau est né le 3 mai 1735 au lieu-dit de Chelfret, et baptisé le 4 à l'église de Cossé-le-Vivien, né de Marie Guyon et de René Rousseau, qui se déclare closier, tisserand mais aussi parfois marchand. René Rousseau est aussi propriétaire de deux petites closeries à Méral, la Chelfret, où il habite, et la Houllerie, qu'il a héritée de sa femme. On est donc assez loin de l'indigence, et à sa mort, en avril 1758, le père laisse une succession se montant à 8116 livres, soit une des plus élevées de l'époque dans la paroisse ${ }^{9}$.

André Rousseau est le dernier de neuf enfants. Chacun d'entre eux est dans une situation relativement stable à la mort de son père, pour ne pas dire prospère pour certains. À l'exception de Pierre, l'avant-dernier fils, encore journalier, mais désigné comme héritier d'une des deux closeries du père, tous sont artisans installés, ou closiers, et les trois filles sont mariées dans ce même milieu. François Rousseau, meunier à Méral, est le plus riche d'entre eux. Tout au long de sa vie, il ne cessera de vendre et d'acheter des terres, qu'il loue ou sous-loue, contractant un nombre impressionnant d'actes notariés. À sa mort, en 1785, il possède sept petites pro-

8. Arch. dép. d'Ille-et-Vilaine, 1 Bm 195, Affaire André Rousseau, Interrogatoire sur la sellette, 21 août 1769 .

9. Arch. dép. de la Mayenne, 3E 27/122, Inventaire René Rousseau, 11-12-13 avril 1758. 
priétés dans les environs de Méral et laisse une succession évaluée au total à 17995 livres $^{10}$.

Quant à André, benjamin de la famille, il perd sa mère à l'âge de huit ans environ, et à seize ans, son père le met en apprentissage chez Jean Durand, maître rouettier ${ }^{11}$ à Saint-Poix, où il reste deux ans ${ }^{12}$. Il ne semble pas avoir appris à écrire, ne signant aucun des documents le concernant, tout comme son père et ses frères. À vingt-deux ans, il s'installe à Méral, où, du moins, il y paye la taille pour la première fois : dix sols. Il a donc déjà une certaine autonomie mais il n'est probablement pas encore installé comme artisan. À vingt-trois ans, son père décède : il n'a pas encore atteint l'âge légal de la majorité, fixé à vingt-cinq ans. Ses frères, sœurs et beaux-frères comparaissent donc devant le sénéchal de la Baronnie de Craon pour désigner son frère Pierre comme tuteur pendant deux ans ${ }^{13}$.

Au cours de la vente et du partage des biens de son père, un mois plus tard, André Rousseau, mineur, est quand même très actif : il acquiert au cours de la vente les biens estimés les plus chers, comme une tasse en argent, le service de couverts en étain, des vêtements, le tout par l'intermédiaire de son frère tuteur, et grâce à l'argent retrouvé chez le père et partagé aussitôt entre les descendants - soit 192 livres chacun. Enfin, il hérite d'une partie de la closerie de la Houllerie à Méral, soit six journées de terre environ, plusieurs vergers et jardins, ainsi que de petits bâtiments de ferme, soit le plus intéressant des huit lots partagés entre les enfants ${ }^{14}$. Car entre temps, il est devenu majeur, et peut donc entrer en possession de biens hérités ${ }^{15}$.

Finalement, il n'entre en possession de ses biens qu'à la Toussaint 1761, et s'installe tout près, dans le bourg de Méral. Il signe un bail pour une maison, se présentant alors comme rouettier habitant à Cossé-le-Vivien ${ }^{16}$. Par la suite, il indiquera, dans d'autres contrats, habiter le village de Grollay, toujours à Méral. En tout cas, il semble déjà être très instable en ce qui concerne ses lieux d'habitation, changeant plusieurs fois en quelques mois. Il tire des revenus de sa closerie en la louant à ferme ${ }^{17}$. Mais rapidement, il rencontre des difficultés financières : il ne parvient pas à rembourser les dettes contractées envers son frère, et doit échelonner ses paiements ${ }^{18}$. De

10. Arch. dép. de la Mayenne, 3E 76/304, Partage des biens François Rousseau, 24 mai 1785 .

11. Fabricant de rouets, servant à filer le lin, principale industrie de la région de Cosséle-Vivien et de Laval à l'époque.

12. Arch. dép. de la Mayenne, 3E 27/115, Brevet d'apprentissage, 16 mai 1751.

13. Arch. dép. de la Mayenne, B 3093, Baronnie de Craon, Registre des sentences de provision, de parentèle..., 14 mars 1758 .

14. Arch. dép. de la Mayenne, 3E 27/124, Partage des biens René Rousseau, 10 avril 1760.

15. Arch. dép. de la Mayenne, 3E 27/124, Liquidation du compte de tutelle, 11 avril 1760.

16. Arch. dép. de la Mayenne, 3E 1/1180, Bail à ferme, 6 novembre 1761.

17. Arch. dép. de la Mayenne, 3E 27/129, Reconnaissance de prisée, 13 février 1762.

18. Arch. dép. de la Mayenne, $3 E$ 27/126, Constitution d'un contrat de rente hypothécaire, 16 septembre 1762 . 
plus, dans sa closerie de la Houllerie, il manque de rentrer en conflit avec son locataire au sujet de la taille des arbres ${ }^{19}$ - cherchant à se procurer du bois pour son travail de rouettier?

Après le mois de juillet 1763, on ne trouve plus aucune trace de lui dans les archives notariales de Méral, jusqu'à son arrestation à Ballot et sa condamnation à Craon en 1764. Il ne paye apparemment pas son amende et est envoyé à Laval pour être attaché à la chaîne de forçats en provenance de Paris et qui se dirige vers Brest, chaque année, au mois de juin. Ayant été condamné au mois de juillet, il doit attendre presque un an dans les geôles de Laval avant que celle-ci ne passe de nouveau. C'est là, très peu de temps avant le passage de la chaîne, dans les prisons de Laval, qu'il semble se décider - poussé par son frère François? - à vendre à celui-ci sa closerie de la Houllerie pour 1100 livres. De cette somme, 300 livres lui sont remises aussitôt, " laquelle somme [...] ledit André Rousseau a déclaré employer à racheter sa liberté des prisons de cette ville où il a été arrêté pour faux saunage ${ }^{20}$ ". Il fait rédiger cet acte dans sa cellule, en présence d'un gabelou et du concierge de la prison, qui lui servent de témoin. Pour autant, il se retrouve quand même sur la chaîne de forçats deux mois plus tard, et arrive au bagne de Brest le 13 juin 1765. Il semble qu'il s'y soit pris trop tard pour régler l'amende : sa peine ayant été convertie depuis près de neuf mois, ses juges n'ont probablement pas voulu accepter ce règlement tardif.

On peut penser que, face au grand nombre de ses dettes, à ses grandes dépenses, aux difficultés éprouvées à gérer son commerce et sa closerie, il était tentant pour lui de résoudre ses problèmes par quelques passages par la Bretagne, son cheval bien chargé de sel, dans le but de remonter le niveau de ses finances. On peut aussi observer une relativement faible cohésion familiale, ses frères et beaux-frères ne s'étant pas précipités pour payer son amende. D'ailleurs, cette condamnation au bagne est vue probablement comme une déchéance pour lui et surtout sa famille, car il ne regagne pas son village d'origine après être revenu de Brest. En effet, il déclare au cours de son deuxième procès devant les Traites et gabelles de Vitré, en 1769, être " journalier de profession à Chemere " (Chéméré-le-Roi? au sud-est de Laval). C'est aussi un signe qu'il est probablement entré définitivement dans la marginalité et la pratique régulière de la contrebande de sel. Il a alors 34 ans. Il est aussi significatif que l'on ne trouve plus aucune trace écrite de lui par la suite, que ce soit chez le notaire ou sur les rôles de taille.

Ce parcours d'André Rousseau est un exemple de faux saunage pratiqué par des couches relativement stables de la société paysanne. Mais cette partie de la société n'est jamais à l'abri d'accidents économiques, de difficultés financières et donc de difficultés sociales. Dans ce milieu de petits artisans, closiers locataires ou même petits propriétaires, on n'est jamais très loin de la "vie fragile ", pouvant très bien se retrouver petit

19. Arch. dép. de la Mayenne, 3E 27/127, Indemnité, 21 juillet 1763.

20. Arch. dép. de la Mayenne, 3E 3/24, Vendition, 27 avril 1765. 
" coq de village " prospère comme son frère François Rousseau, tout aussi bien que journalier mendiant et petit délinquant à ses heures.

\section{Pierre Boisramé}

Tout comme André Rousseau, ce n'est qu'une partie de la vie de Pierre Boisramé qui se dévoile clairement au vu des archives retrouvées. Pour autant, c'est une tranche de vie intéressante car c'est le seul pour lequel des traces ont pu être retrouvées après son retour du bagne.

Pierre Boisramé est issu d'une famille de très modestes tisserands de Cossé-le-Vivien. Il est né le 26 août 1734, de Marie Beaujan et de Louis Boisramé. On lui connaît deux frères, beaucoup plus âgés que lui, Louis et Jean, eux aussi tisserands ou closiers à Cossé-le-Vivien. Son père décède rapidement, et Pierre Boisramé se retrouve orphelin probablement dès l'âge de 12 ans. Sa mère se remarie avec un poupelier ${ }^{21}$ de Cossé-le-Vivien, Mathurin Beasse. Son beau-père l'envoie rapidement en apprentissage, dès l'âge de 13 ans, chez le marchand tissier René Boisramé, à la Touchebaron, toujours à Cossé-le-Vivien pour une période de deux ans ${ }^{22}$. Il ne semble pas avoir appris à lire ou écrire, ne signant aucun des documents le concernant.

La seconde trace que l'on ait de lui par la suite est le procès-verbal des gabelous qui l'arrêtent, onze ans plus tard, à l'âge de vingt-quatre ans. Contrairement à ce qu'auraient pu faire penser ses origines modestes, il est arrêté au sein d'une grande bande de piqueurs, des faux sauniers à cheval montés, tous armés de masserottes et autres bâtons, se déplaçant en grand nombre pour effrayer les employés des Fermes. Ce mode de contrebande est traditionnellement le fait de marchands et de voituriers, professionnels de la route et aux moyens suffisants pour s'acheter un bon cheval et des grandes quantités de $\operatorname{sel}^{23}$. On peut s'étonner de retrouver ainsi un petit tisserand : est-ce justement la pratique régulière du faux saunage qui lui en aurait donné les moyens? Il a beau être arrêté dans un attroupement de vingt-cinq contrebandiers, avec une arme, et après s'en être servi contre les employés, la commission de Saumur, clémente, ne le condamne que pour simple faux saunage à cheval, à 300 livres d'amende. Pour autant, il ne les paie pas, et est envoyé au bagne de Brest pour trois ans ${ }^{24}$.

Il est libéré du bagne le 24 avril 1762. Dès le mois de mai suivant, il est rentré chez lui : en effet, entre temps, devenu majeur, il peut réclamer sa part de l'héritage de son père à sa mère et à ses frères. Il vend ainsi la mai-

21. Petit artisan chargé de transformer les tiges de lin en filasse pour que celle-ci soit ensuite filée, puis tissée.

22. Arch. dép. de la Mayenne, 3E 39/247, Brevet d'apprentissage, 6 février 1747.

23. Cf. Huvet-Martinet, Micheline "Faux saunage et faux sauniers... ", art. cit., 1978, 4, p. 573-594 sur les différents types de faux saunage.

24. AN, Z 1A/1110, affaire Pierre Boisramé, 24 mai 1759.

25. Arch. dép. de la Mayenne, 3E 27/126, Contrat de vente, 31 mai 1762. 
son, propriété de son père, dans le bourg de Méral, pour toucher le tiers du revenu de la vente, le 31 mai $^{25}$.

Il est alors âgé de vingt-huit ans, et semble chercher à se stabiliser rapidement, signe peut-être qu'il veut couper avec sa vie de faux saunier. On trouve très rarement de jeunes faux sauniers mariés : or, il se marie le 31 janvier 1764, avec Jacquine Madiot, fille, comme lui, d'un petit tisserand de Cossé-le-Vivien. Ses frères sont ses témoins. Un contrat de mariage est signé à cette occasion : Pierre Boisramé apporte 30 livres " qu'il a en argent provenant de son travail " et sa femme apporte 95 livres de $\operatorname{dot}^{26}$. De même, quelques mois plus tard, alors qu'il habitait au hameau de la Frenouze, chez - ou à proximité de chez - son frère aîné Louis, closier, il décide de louer une maison à la Bapaume, un autre gros village de la paroisse de Cossé-leVivien. Il loue cette maison à ferme avec un jardin, pour cinq années et pour 25 livres par $a^{27}$. À partir de cette même année, il est enrôlé et paie la taille pour la première fois dans la paroisse, même si celle-ci ne s'élève qu'à 37 sols - et 25 sols de capitation. Ce bail à la Bapaume ne sera pas renouvelé au bout des cinq ans, et Pierre Boisramé retourne par la suite, en 1769, habiter à la Frenouze près de son frère, avec sa famille. Celle-ci s'est considérablement agrandie. Dés 1764, naît sa première fille et neuf autres enfants suivront ${ }^{28}$, jusqu'à la naissance du dernier en 1777 , au bout de quatorze ans de mariage avec Jacquine Madiot, ce qui est considéré comme exceptionnel, même par le curé de Cossé-le-Vivien ${ }^{29}$.

Tous ces éléments de la vie de Pierre Boisramé sont des gages de stabilité, d'une "vie rangée ", si on peut dire, car on ne retrouve pas ces informations pour les autres faux sauniers, chez ceux qui ne semblent pas avoir cessé de pratiquer la contrebande, au vu de leurs arrestations. On peut y voir peut-être la volonté de couper avec une jeunesse plus aventureuse et mouvementée. Mais pour autant, on ne peut être certain qu'il ait coupé les ponts complètement avec le milieu des faux sauniers. En effet, certains documents pourraient même faire penser le contraire.

Entre 1774 et 1779 , le conseil paroissial responsable des montants de la taille pour chaque paroissien décide, en ce qui concerne Pierre Boisramé, " que sa taxe soit augmentée pour les terres qu'exploitait Gabriel Lendemain audit lieu qu'il exploite ${ }^{30}$ ". En clair, Pierre Boisramé cultive temporairement les terres de Gabriel Lendemain, en l'absence de celui-ci. Ainsi, alors qu'il avait payé 40 sols de taille en 1774, il paye pendant cinq ans, de 1775 à 1779, trois livres, pour revenir à vingt sols par la suite. Or, au

26. Arch. dép. de la Mayenne, 3E 39/261, Contrat de mariage, 5 janvier 1764 .

27. Arch. dép. de la Mayenne, 3E 39/261, Bail à ferme, 5 septembre 1764.

28. Jacquine, Julien et Pierre, Marie-Jacquine, Pierre, Louis, Jean-Pierre, Pierre-Jacques, Jacquine-Louise et Jeanne. Il faut noter que je ne sais pas s'ils ont tous survécu, n'ayant pas pu relever les actes de sépulture.

29. Arch. dép. de la Mayenne, 5 Mi 739, registres paroissiaux de Cossé-le-Vivien, 22 août 1777.

30. Arch. dép. de la Mayenne, C 235, Rôles de taille de la paroisse de Cossé-le-Vivien, délibération de 1774. 
même moment, Gabriel Lendemain, en compagnie de trois complices, se fait arrêter pour faux saunage à col, au village de la Normandière, à Cosséle-Vivien, et est envoyé aux galères pour récidive pour six années ${ }^{31}$. Déjà, en 1771, Pierre Boisramé avait repris et exploité les terres de Jean Lecerf, cousin par alliance de Gabriel Lendemain, pendant trois ans, ce qui l'avait obligé à payer trois livres supplémentaires pour le règlement de la taille. Même si aucune procédure contre ledit Lecerf n'a pu être retrouvée ${ }^{32}$.

Dans ce milieu de petits paysans, journaliers, tisserands, fileurs, à la frontière de la Bretagne, il semble que l'on reste toujours dans un milieu pratiquant plus ou moins occasionnellement le faux saunage, pour arrondir un maigre salaire en période de crise textile ou pour faciliter la soudure, à l'occasion. Ce qui a pu tout à fait être le cas de Pierre Boisramé, mais cette fois-ci sans se faire prendre, semble-t-il, puisque nous n'en avons pas la preuve.

En 1779, sa femme décède à l'âge de quarante-cinq ans, en période de forte mortalité et probablement d'épidémie dans la paroisse ${ }^{33}$. En 1782 , Pierre Boisramé disparaît des rôles de taille, et dès lors, ne laisse plus aucune trace de lui dans les archives : il n'apparaît même pas dans la liste des pauvres de la paroisse, ni dans les dérôlables, ni dans les sépultures des registres paroissiaux de Cossé-le-Vivien. Il est alors âgé de quarantesept ans. S'est-il remarié à l'extérieur de la paroisse? Est-il retourné dans la marginalité totale d'un faux saunier?

\section{Apports d'une telle recherche dans la connaissance du faux saunage}

Il est difficile de donner des conclusions générales à partir d'un échantillon aussi réduit et avec des informations aussi lacunaires, néanmoins, comme il a été dit plus haut, cette étude permet d'avoir un autre point de vue, apportant ainsi des nuances aux connaissances précédentes sur le faux saunage. Ces apports concernent avant tout la place du faux saunier dans la société de l'époque.

Ils permettent tout d'abord de préciser les conditions socio-économiques du faux saunage : la contrebande de sel apparaît comme l'un des rouages du système de marginalisation et de l'entrée dans la pauvreté. Face à des sources aussi lacunaires, il est bien difficile de distinguer des motivations individuelles à la pratique de la contrebande, mais les sources seraient-elles plus complètes, l'explication ne serait pas beaucoup plus

31. Arch. dép. de la Mayenne, B 3442, Affaire François Lemoyne, Étienne Rousseau, Pierre Cormier, et Gabriel Lendemain dit Taupin, 17 mai 1775. Gabriel Lendemain avait déjà été condamné par le Grenier à sel de Laval le 13 mai 1772.

32. Arch. dép. de la Mayenne, C 235, Délibération du conseil paroissial du 11 décembre $1771:$ « [... Pierre Boisramé tissier et closier à la Frenouze, que sa taxe soit augmentée pour raison des erbages et enseptemances de la closerie qu'exploitoit ledit Jean Lecerf [...]."

33. Arch. dép. de la Mayenne, 5 mi 739, registres paroissiaux de Cossé-le-Vivien, 30 octobre 1779 . 
facile. En réalité, dans les différents parcours ici décrits, il faut chercher des stratégies plus que des motivations. Ainsi, le petit journalier, le fileur, le tisserand sans ouvrage, ont toujours la possibilité, pour assurer leur survie et celle de leur famille, de faire quelques passages de sel de l'autre côté de la frontière : cela demande peu d'investissements et cela peut rapporter beaucoup plus que leurs revenus habituels. Mais cela comporte aussi des risques pour le candidat au faux saunage : celui de se faire arrêter et surtout de perdre la petite situation qu'il possédait, la petite stabilité qu'il était parvenu à maintenir au sein de sa famille ou de son village, stabilité observable dans plusieurs sources telles les rôles de taille. Car il est très difficile de revenir par la suite à une vie respectable, inscrite dans la vie officielle du village, comme on a pu le voir avec André Rousseau ou Pierre Boisramé. À l'inverse, un individu ne peut rester en permanence dans la clandestinité du faux saunier : il revient toujours de temps en temps vers la normalité, c'est-à-dire qu'il réapparaît dans les sources.

Dans les relations sociales ensuite. La contrebande ne peut fonctionner correctement sans un réseau de relations bien établi. Outre le simple passeur qui est chargé du transport de la marchandise, à l'endroit le plus difficile, c'est-à-dire la frontière et les quelques kilomètres aux alentours, il faut un fournisseur, pour apporter la marchandise au plus près du lieu interdit, puis un revendeur pour l'écouler, une fois la frontière passée. Mais ce petit noyau du réseau ne suffit pas. Dans une campagne si densément peuplée, où tout se sait et où tout le monde s'observe, il faut un réseau de complaisances beaucoup plus élargi pour ne pas risquer la dénonciation. C'est en effet parce que tout le monde préfère le faux saunier au gabelou, pour de multiples raisons que la contrebande de sel à si grande échelle peut exister à la frontière entre la Bretagne, le Maine et l'Anjou au XVIII ${ }^{\mathrm{e}}$ siècle.

Ce réseau peut à la fois exister à un petit niveau, c'est-à-dire au niveau des connaissances proches, que l'on côtoie tous les jours, mais ces solidarités familiales ne semblent pas déterminantes pour l'organisation du trafic. D'une part car les familles auxquelles ces individus appartiennent sont souvent démembrées, avec un très grand nombre d'orphelins comme on a pu le voir par exemple ici avec André Rousseau et Pierre Boisramé, et d'autre part car il ne semble pas exister, pour ce qui a été observé, de modèle de familles organisées en clans, avec des relations de solidarité, d'autorité, d'allégeance, au sein d'une structure extrêmement soudée qu'est la famille élargie. Ce modèle de " mafia " repéré dans les milieux délinquants d'autres régions, du Sud de la France et de l'Europe, n'est pas présent dans la contrebande de l'Ouest de la France. Mais les solidarités avec les contrebandiers du sel peuvent exister aussi au niveau de la communauté tout entière qui voit passer des faux sauniers devant elle tous les jours, qui les soutient et qui en tire profit. La contrebande est une pratique courante, quasiment acceptée par tous puisque bon nombre des gens des classes populaires de cette région sont susceptibles d'en faire un jour ou l'autre. On connaît très peu d'exemples d'attitudes hostiles envers les passeurs de 
faux sel, surtout à proximité de la frontière de Bretagne. Mais cette indifférence voire cette complaisance envers le faux saunage change complètement lorsque ceux-ci s'en prennent à leurs biens ou à eux mêmes, et non plus aux privilèges de l'autorité lointaine et abhorrée qu'est la Ferme.

L'historien a tendance, de part sa base de travail - l'archive - à privilégier une approche macro-analytique des phénomènes qu'il étudie. En effet, ces archives sont le produit d'institutions et la facilité pousse à analyser ces archives par le biais de ces institutions et des grands ensembles de données en général. Ce type d'approche a produit évidemment d'énormes apports à la connaissance historique, puisque cette étude elle-même s'est appuyée beaucoup sur ces études macro-analytiques du faux saunage. Mais on ne peut se contenter seulement d'une telle approche. En outre, lorsque le domaine de recherche est déjà bien connu et balisé, comme c'est le cas de la contrebande de sel à la frontière de Bretagne, aborder le sujet " par le bas " permet de renouveler l'étude et de nuancer les conclusions déjà apportées par ces précédentes études.

De telles recherches pour des populations faux saunières récidivistes aussi mobiles et indigentes se révèlent effectivement bien difficiles, mais néanmoins parfois possibles dans les zones de forte concentration de faux saunage. Et une étude à partir du nom se révèle tout de même possible dans ces sources pour des individus laissant un peu plus de traces " volontaires " dans les archives. Enfin, cette étude a voulu aussi montrer que cette recherche pouvait intégrer des sources judiciaires comme point de départ à la recherche de destins personnels de criminels, enrichie par d'autres sources, dans le but de comprendre le trajet d'un individu retrouvé dans les archives judiciaires. 


\section{RESUME}

Plusieurs recherches ont déjà porté sur les archives de procès de contrebande de sel, mais aucune ne s'est intéressée à des cas individuels de fauxsauniers. Pourtant, il s'agit ici d'individus susceptibles d'être retrouvés dans plusieurs procès, dans les registres du bagne de Brest et dans des archives très localisées, comme c'est le cas à la frontière de la Bretagne. Mais la recherche de ces individus très mobiles est particulièrement complexe. Toutefois, les résultats obtenus sont éclairants concernant les parcours personnels de populations rurales modestes. Ils montrent des individus toujours à la limite de la marginalité, mais bénéficiant de soutiens et d'une cohésion sans faille face aux agents de la Ferme des gabelles.

\footnotetext{
ABSTRACT

In search of salt smugglers at the borders of Maine, Anjou and Brittany at the end of the $18^{\text {th }}$ Century

There have been several researches in the archives of lawsuits about salt smugglers, but none focused on individual cases of false workers in saltworks. However, it concerns here individuals likely to be met again in several trials in the registers of the penal colony in Brest or very localised archives, as it is the case on the border of Brittany. But the research of these very mobile individuals is particularly complex. However, the results we have got give interesting information concerning the personal itineraries of modest rural populations. They show individuals always on the verge of marginality, but benefitting from the endorsement of the rural society and thorougly united against the salt tax-collector agents.
} 
